\title{
Facilidades e dificuldades dos enfermeiros no cuidar da alimentação infantil na atenção básica
} Advantages and Problems of nurses in care of infant feeding in primary care

\author{
Mayara Águida Porfírio Moura* \\ Silvana Santiago da Rocha** \\ Diana Lúcia Moura Pinho*** \\ Dirce Guilhem **
}

\begin{abstract}
Resumo
No Brasil, a ampliação da rede de atenção básica nos últimos anos, e as mudanças ocorridas na situação nutricional infantil, reflete no manejo do cuidado de enfermagem à criança. O objetivo do estudo foi apreender facilidades e dificuldades no cuidado de enfermagem, relacionado à alimentação infantil no cotidiano do enfermeiro na rede de atenção básica de Teresina-PI. Trata-se de um estudo com abordagem qualitativa, pautado na história oral temática. Entrevistou-se 16 enfermeiros do quadro efetivos da rede de atenção básica, utilizando-se roteiro semiestruturado. As entrevistas foram gravadas e transcritas, prosseguindo com análise em história oral. Nos resultados e discussão, identificou-se que as facilidades no cuidado de enfermagem na alimentação infantil envolvem as categorias: 1) Criança e família; 2) Enfermeiro; e 3) Unidade de atenção básica. Quanto às dificuldades, estas se concentram no universo da criança englobando fatores sociais, culturais e econômicos de seu contexto. Conclui-se que as facilidades relatadas podem constituir-se em alavanca para o cuidado de enfermagem e as dificuldades representam entraves a serem superadas pelos enfermeiros, e explorados em outros estudos. Evidencia-se com o estudo, a importância da alimentação e do cuidado de Enfermagem para a promoção da saúde da criança e de sua qualidade de vida.
\end{abstract}

Palavras-chave: Enfermagem Pediátrica. Cuidado de Enfermagem. Atenção Primária à Saúde. Alimentação Infantil.

\begin{abstract}
In Brazil, the expansion of the primary care network in recent years and the changes in children's nutritional status reflected in new advantages and difficulties in children's nursing care. The aim of the study was to report the strengths and difficulties in nursing care related to infant feeding in the nursing in the routine day in primary care network of Teresina-PI. As regards methodology, we opted for a qualitative study, based on thematic oral history. We interviewed 16 nurses of effective primary care network using a semi-structured interview. The interviews were recorded and transcribed, continuing analysis of oral history. In the results and discussion, it was identified that the advantages in nursing care on infant feeding involve the following categories: 1) Child and Family; 2) Nurse; and 3) Primary Care Unit. As for the difficulties, these focus on children's universe, encompassing social, cultural and economic factors of context. We conclude that the reported strengths can be constituted in a lever for nursing care and the difficulties pose obstacles to be overcome by nurses and are explored in other studies. It is evident to the study the importance of nutrition and nursing care for the promotion of children's health and their quality of life.
\end{abstract}

Keywords: Pediatric Nursing. Nursing Care. Primary Health Care. Baby Food.

DOI: $10.15343 / 0104-7809.20153902231238$

* Departamento de Enfermagem. Teresina, Piauí, Brasil. E-mail: mai_moura@hotmail.com.

** Departamento de Enfermagem, Teresina-PI, Brasil.

*** Universidade de Brasília, Brasília-DF, Brasil.

As autoras declaram não haver conflitos de interesse. 


\section{INTRODUÇÃO}

Nos últimos anos no Brasil, importantes alterações têm ocorrido, em relação à situação nutricional infantil. Corroborando com a expansão da rede de atenção básica brasileira, na perspectiva de uma compreensão mais abrangente sobre as facilidades e dificuldades emergidos no cotidiano da assistência à saúde da criança, sobre o cuidado de enfermagem e a alimentação infantil no contexto da atenção básica.

A infância é uma fase da vida caracterizada por mudanças significativas representando um momento fisiologicamente vulnerável. Alimentação e nutrição adequadas, são fundamentais para garantir a manutenção da saúde, favorecendo o crescimento e desenvolvimento da criança ${ }^{1-3}$.

Observa-se que o Brasil acompanha as tendências internacionais em relação à transição nutricional que é caracterizada pela diminuição da desnutrição (contudo, ainda tem merecido atenção em condições socioeconômicas menos favorecidas), neste contexto a obesidade emerge como epidemia. Os problemas nutricionais influenciam os agravos de saúde e a mortalidade infantil ${ }^{4-6}$.

Com base nesses fatos, cabe ao profissional da saúde identificar problemas alimentares e nutricionais da criança, a fim de estabelecer estratégias de cuidados que possibilitem a diminuição de morbidades e mortalidades relacionadas ao estado nutricional, contribuindo com crescimento e desenvolvimento adequados para a faixa etária e formação de hábitos alimentares saudáveis ${ }^{7}$.

Em 21 de outubro de 2011, o Ministério da Saúde, por meio da portaria $N^{\circ} 2.488$, aprovou a Política Nacional da Atenção Básica (PNAB), com a revisão de diretrizes e normas organizacionais para a atenção básica e em especial para a Estratégia Saúde da Família. A nova portaria sobre a PNAB não revoga a anterior ( $\left.N^{\circ} 648 / 2006\right)$, estabelece apenas que o Ministério da Saúde por meio das secretarias fica encarregado de publicar guias/manuais que orientem tal política, definindo também os recursos orçamentários

destinados a onerar com tais gastos, garantindo assim sua continuidade ${ }^{8-9}$.

O Piauí é o estado Brasileiro que possui maior cobertura populacional da Estratégia Saúde da Família (ESF), iniciada em 1994 teve uma ampliação significativa a partir de 2000, e em 2010 abrange cerca de 1.100 equipes Saúde da Família atendendo três milhões de pessoas nos serviços de atenção básica ${ }^{10}$. Diante dessa realidade, em 2012 a Prefeitura Municipal de Teresina, por meio da Fundação Municipal de Saúde, publicou o "Protocolo de Enfermagem na atenção básica de saúde". Que tem como objetivo guiar o profissional no intuito de contribuir para a redução dos índices de morbidade e mortalidade das crianças, propondo uma assistência de enfermagem qualificada e planejada. O protocolo define a Consulta de Enfermagem à criança numa avaliação sistemática e engloba: crescimento e desenvolvimento, nutrição, imunização, cuidados e higiene, entre outras avaliações ${ }^{11}$.

A dinâmica da assistência de enfermagem à criança na atenção básica de Teresina-PI, é operacionalizada por meio da consulta de enfermagem seguindo o proposto pela Política Nacional da Atenção Básica, focada nas ações de promoção da saúde e na prevenção de doenças. Na perspectiva da criança, este cuidado é desenvolvido na visita domiciliar na Primeira Semana de Saúde Integral da mãe e da criança, nas consultas de puericultura, e na participação do enfermeiro no programa de auxílio financeiro governamental. O enfermeiro contribui para a promoção da qualidade de vida da criança articulando saberes e práticas do cuidado, visando garantir condições mais saudáveis para a criança ${ }^{12}$.

Desta forma, o objetivo desta pesquisa foi apreender, por meio das falas dos enfermeiros, facilidades e dificuldades no cuidado de enfermagem, relacionadas à alimentação infantil em seu cotidiano no contexto da atenção básica à saúde no município de Teresina/Piauí. 


\section{MÉTODO}

Optou-se por um estudo de abordagem qualitativa, do tipo história oral temática. Esta se articula como um conjunto de procedimentos planejados, obtidos por meio de diálogos gravados e posteriormente transcritos, que registram a percepção da vida social ou coletiva com a finalidade de constituir fontes ou documentos ${ }^{13}$.

Participaram do estudo, 16 enfermeiros do quadro de efetivos das unidades de atenção básica da zona urbana de Teresina que atenderam aos seguintes critérios de inclusão: ter no mínimo seis meses de experiência na estratégia Saúde da Família; desenvolver ações direcionadas à saúde da criança. Em cada unidade básica incluída no estudo, optou-se por realizar a entrevista com um enfermeiro que tivesse disponibilidade no momento da coleta de dados, seguindo assim, o critério de seleção de amostra do tipo incidental.

Na história oral temática a definição do número de participantes a serem entrevistados em uma pesquisa deve ser significativa para viabilizar a generalização dos resultados. Assim, a partir do momento em que as entrevistas se tornam repetitivas, ocorre o ponto de saturação, indicando que a coleta dos dados deve ser finalizada ${ }^{13}$.

$\mathrm{Na}$ abordagem aos participantes, foram explicitados os objetivos do estudo e solicitado à participação voluntaria e o consentimento oficializados por meio da assinatura do Termo de Consentimento Livre e Esclarecido. Todos os participantes tiveram direito à privacidade e a confidencialidade sobre a origem dos dados. Para isto, os nomes foram substituídos pelo termo "enfermeiro" numerados de 1 a 16, não levando em consideração a ordem de realização das entrevistas.

Os cenários da pesquisa foram 16 unidades básicas de saúde da estratégia Saúde da Família pertencentes à rede de atenção básica, todas situadas na área urbana de Teresina-PI, localizadas em três grandes áreas: norte, sul e leste-sudeste. A inclusão das unidades no estudo foi realizada por meio de sorteio das unidades básicas por grande área da cidade, contemplando assim todas as áreas, seguindo a ordem alfabética (norte, leste-sudeste e sul).

Para a produção dos dados foi utilizada a técnica de entrevista com roteiro semiestruturado e gravação em áudio, com equipamento tecnologicamente adequado. Após a coleta dos dados, as entrevistas foram transcritas na íntegra.

Para o tratamento dos dados, foi utilizada a análise em história oral, trata-se de um procedimento metodológico planejado. Este procedimento consiste na definição de temas relevantes ou núcleos de sentidos e não na divisão racional do conteúdo das entrevistas em conjunto ${ }^{13}$.

Como tema relevante ou núcleo de sentidos, optou-se por descrever os resultados e discussão em duas partes: 1) O relato das facilidades pelos enfermeiros no cuidado com a alimentação infantil e 2) As dificuldades no cuidado com a alimentação infantil.

Este estudo foi aprovado pela Fundação Municipal de Saúde de Teresina-PI (órgão ao qual está vinculado as unidades básicas de saúde da atenção básica) e pelo Comitê de Ética na Pesquisa (CEP) da Universidade Federal do Piauí (UFPI), sob o CAAE nº 0291.0.045.000-10.

\section{RESULTADOS E DISCUSSÃO}

\section{1) 0 relato das facilidades relatadas pelos enfermeiros no cuidado com a alimentação infantil}

No cuidado à criança na atenção básica, pode-se encontrar situações que possibilitem manejar esta atividade da alimentação infantil com mais facilidade, por meio do conhecimento de fatores condicionantes relacionados à criança e sua família; ao enfermeiro ou a estrutura e processo de trabalho na unidade básica de saúde, representando verdadeiras alavancas potencializadoras do cuidado.

No tocante as facilidades identificadas e relacionadas à criança e sua família, a figura materna se apresenta como principal responsável pelo cuidado com a criança e sua alimentação. Sob esta perspectiva, os seguintes depoimentos revelam tal potencialidade:

"Então a gente orienta como deve ser a alimentação saudável, mas percebemos que 
na prática muitas mães são cuidadosas e seguem as orientações" (Enfermeiro 03).

"Entretanto, as mães de minha área são muito acessíveis, sempre acolhem o que eu falo, digo, realmente acolhem muito bem" (Enfermeiro 13).

"Uma parte das mães é acessível, isso facilita o trabalho, elas colaboram, a maioria das mães procura mais no primeiro ano de vida, principalmente por conta das vacinas, ai aproveitamos para orientar" (Enfermeiro 14). A mãe é considerada um exemplo de cuidado, irradiam o cuidado privilegiadamente. Distinguida por ser o primeiro continente que uma criança conhece, incorpora bem mais que funções maternas e representa um modo-de-ser que se compõe de dimensões de mulher-mãe com seu corpo, psique e espírito. Esse corpo envolve uma dimensão complexa de sentimentos incorporando ações que fortalecem o sentido de provedora de alimento e aprendiz dos serviços da maternidade ${ }^{14-15}$.

Percebe-se a relação maternidade, alimentação e recém-nascido como fundamental para o estabelecimento do vínculo, proporcionando a aproximação da mãe como principal cuidadora, provedora da alimentação básica deste ser.

Nesse sentido, um depoimento relata situações que favorecem o acolhimento das mães em relação às orientações e os cuidados realizados pelos enfermeiros:

"Eu acho que o que realmente facilita é se começarmos a trabalhar logo na primeira semana, porque assim as orientações vão mudando mês a mês, e a mãe vai seguindo. E também quando começamos a trabalhar no pré-natal, isso facilita demais porque a mãe segue ainda mais as nossas orientações" (Enfermeiro 11).

Tais situações, revelam estratégias de cuidado que possibilitam estabelecer vínculo de continuidade, e se mostra muito positiva tanto para o enfermeiro como para o binômio mãe e filho. É na primeira semana que surgem dúvidas, inseguranças e questionamentos das famílias, sendo esse período precioso para a atuação da equipe, de modo que seus profissionais possam ser reconhecidos como um ponto de apoio para a superação dessa etapa. Essa é uma estratégia com foco no binômio mãe-bebê ressaltada na "Primeira Semana Saúde Integral"16.

No contexto da criança e de sua família, outro fator condicionante que facilita a prática do enfermeiro no cuidado à criança está relacionado à questão financeira que possibilita a família condição mínima para que haja saúde entre seus membros e, em especial, as crianças.

"Já trabalhamos em bairro mais carente, mas graças a Deus este bairro tem pessoas que tem o mínimo de recursos financeiros, tendo mais acesso aos alimentos" (Enfermeiro 08). "O que facilita é a condição financeira, porque você pode pedir para que ela compre alimentos, medicamentos caros e mais completos que o SUS não oferece, como polivitamínico, elas não se restringem ao ferro da unidade" (Enfermeiro 15).

Em contrapartida, em situações econômicas desfavoráveis, as mulheres se deparam com a necessidade de trabalhar e este condicionante pode ser um fator de risco para a nutrição da criança. Situações de pobreza levam a dificuldades com o provimento do cuidado com a alimentação da criança, muitas vezes, não compensado pela renda gerada, repercutindo em prejuízo com a nutrição da criança ${ }^{17}$.

$\mathrm{Na}$ dimensão profissional os aspectos que facilitam o cuidado com a alimentação infantil, foram apreendidos a partir dos estratos dos depoimentos a seguir, nos quais os enfermeiros se colocam como sujeitos ativos e envolvidos no processo, o que implica em uma maior participação.

"Eu sempre gosto de colocar a minha situação, com os meus filhos, o que eu pude evitar, eu fiz, você tem que supervisionar, colocou também que é importante" (Enfermeiro 08).

"A integração da equipe com o mesmo objetivo facilita a transmissão de informações, e assim efetiva uma educação em saúde" (Enfermeiro 10).

São relatos que retratam de maneira diferente a prática da enfermagem que contribui como 
instrumento facilitador. O modo-de-ser-no-mundo, conduz o ser humano à adaptação do meio ao seu desejo e à conformação do desejo ao meio em busca do "habitat" que proporcione a evolução conscientemente ${ }^{14}$.

Percebe-se que o envolvimento dos demais integrantes da equipe de saúde em uma intencionalidade de mudanças de atitude, por meio do compartilhamento de informação, proporciona reflexão e expressão de ideias e sentimentos $^{18}$. Com isso, observa-se uma dimensão mais complexa, na qual o profissional está inserido e envolvido não apenas pela prática dirigida à criança, e a sua família, mas também, com a equipe multiprofissional, a fim de potencializar suas ações, compreendendo um atendimento mais integral por meio da comunicação.

$\mathrm{Na}$ dimensão do campo de trabalho, percebe-se que as unidades básicas de saúde são locais vistos como ação preventiva pelos usuários, as quais ao longo do tempo incorporaram e passaram a entender os significados da promoção da saúde e da prevenção de doenças. Outros aspectos relacionados, dizem respeito à acessibilidade dos usuários aos serviços de saúde, como ressaltam os participantes 09 e 15:

"Uma coisa que favorece a gente implementar é que as mães não estão mais trazendo as crianças somente quando elas estão doentes, o sentido da puericultura em relação à prevenção realmente está acontecendo, trabaIhando o acompanhamento do crescimento e desenvolvimento, trazendo a promoção da saúde para essas crianças" (Enfermeiro 09). "O que facilita é a localização da unidade de saúde, porque eu observo que a demanda é grande porque a unidade esta perto das pessoas. A minha colega de outra equipe não experimenta isso por que a população dela precisa pegar ônibus, e eles são carentes. Então a unidade está dentro da minha área, isso facilita muito o trabalho" (Enfermeiro 15).

A atenção básica é caracterizada por ações resolutivas de baixa complexidade com vistas a proporcionar a integralidade das ações de saúde por meio de uma assistência qualificada.
A estratégia Saúde da Família é considerada uma alavanca de mudança para os sistemas de saúde brasileiros ${ }^{19}$.

Ressalta-se então, a importância da acessibilidade dos usuários aos serviços de saúde, construindo-se significados atribuídos às unidades básicas de saúde e garantindo-se as ações que visam à busca de um conceito mais amplo de saúde. Incluem-se, desta forma, as ações de prevenção de doenças e de promoção da saúde, corroborando como instrumentos que representam os avanços na aplicabilidade do cuidado de enfermagem à criança.

\section{2) Dificuldades percebidas no cuidado com a alimentação infantil}

Constatou-se pelos relatos dos participantes, que ainda há dificuldades no cotidiano desses enfermeiros, relacionados à criança e à família, em seus aspectos sociais, culturais e econômicos. Na dimensão social, destaca-se o papel da mãe e suas implicações no cuidado à criança. A partir desse contexto, os depoimentos apontam para a idade materna, o tipo de trabalho ou inexperiência da mãe como fatores que podem repercutir na transferência da responsabilidade do cuidar, sendo que os principais substitutivos desse cuidar são representados pela avó, tia, babá e vizinhos.

"O que dificulta são as mães, mães novas demais que não querem ter trabalho, taca logo o leite e não quer dar o peito, entendeu? Ou aquelas que têm interferência muito forte das avós, que querem botar muita comida e fazer diferente" (Enfermeiro 02).

"Tem muita mãe que simplesmente não quer ouvir palestras, orientações. Às vezes, elas mandam os filhos com os vizinhos, mas elas mesmas não vêm" (Enfermeiro 12). "O que dificulta é os pais deixarem o filho com a babá, vizinha ou a avó que não têm tempo e destreza para estar colocando a forma de alimentação como prazer, ela rejeita e vai sofrer pra comer, isso dificulta demais. Além da facilidade de comprar guloseimas que as crianças vão ficar quietas e não vão encher o saco dele." (Enfermeiro 15). 
A literatura destaca que existem pessoas que cuidam menos do que deveriam, suas ações são desenvolvidas de forma incompletas não acompanhando a perspectiva existente ou, em alguns casos, assumem muitas responsabilidades, deixando de alcançar os objetivos propostos. Outras não dispensam o cuidado com o empenho merecido, resultando em desordem, caos e confusão ${ }^{14}$.

Estudos sobre negligência e abandono em menores de quinze anos, revela que o perfil materno nesses casos está relacionado principalmente com a idade materna inferior a 24 anos, ou como considerada pelos autores "mãe muito jovem" ou sem maturidade para a maternidade $^{20}$. Condições desfavoráveis para a mulher podem causar prejuízos ao cuidado à criança neste momento de formação de vínculos.

Neste contexto, seria interessante que os enfermeiros pudessem identificar e compreender as dificuldades singulares para estas mães desenvolverem as ações, sem estigmatiza-las, apresentando assim, estratégias que possa contribuir para equacionar a problemática.

Os depoimentos revelaram valores e significados culturais, materializando-se nas seguintes situações: a concepção materna ou familiar de acreditar que a criança está saudável quando a mesma se encontra com peso acima do normal e a preocupação de haver deformidades em seu corpo.

"Às vezes as mães dizem que as crianças estão com baixo peso, quando na verdade não estão, existe uma cultura de que criança gordinha é criança saudável" (Enfermeiro 04). "A família, os avós, às vezes acham que a criança não está engordando muito, e aí dá leite, e até mesmo as mães não acreditam e quando fazemos uma visita descobrimos que ela já está dando outros alimentos e escondendo da equipe" (Enfermeiro 10). "É aquela história que se amamentar o seio vai amolecer, mas isso já está melhorando muito. É a cultura mesmo, de dar chá, de dar isso ou aquilo, e é por isso que desde a gravidez que tentamos trabalhar a mãe, falamos do custo benefício" (Enfermeiro 08).
Em relação à alimentação da criança, percebe-se que a mãe muitas vezes, não acompanha as orientações oferecidas pelos profissionais, muitas vezes por receio de que os profissionais possam percebê-las como descuidadas com a criança. Adota o uso de outros alimentos para favorecer o ganho de peso da criança, o que é reflexo de uma concepção errônea de saúde.

Nesse contexto, existem evidências que apontam para o despreparo das nutrizes sobre técnicas e orientações corretas sobre o aleitamento materno apontando para possibilidades de atuação principalmente dos enfermeiros ainda no pré-natal ${ }^{21}$.

Estudos sobre o desmame precoce, apontaram que as mães possuíam suas crenças, tradições e valores ancorados em experiências de outras pessoas. Observou-se, ainda, a relação gordura e saúde com o valor do estereótipo "bebê gordinho" como determinante no julgamento de estar ou não cuidando satisfatoriamente da criança ${ }^{22}$.

Nesse sentido, observa-se a prática errônea relacionada à nutrição infantil com a valorização da criança obesa ou com sobrepeso, o que na realidade pode significar a existência de problemas em virtude das possibilidades de morbidade e mortalidade relacionadas à obesidade infantil.

Ainda nesse momento, as mães percebem as modificações ocorridas com a maternidade em relação ao seu corpo, e esta preocupação incorpora significados culturais à luz da interpretação do senso comum, como o exposto pelo participante 08 .

O dito "peito caído" representa uma construção social das consequências anatômicas adquiridas com o ato da amamentação, conduzindo a uma percepção negativa do corpo da mulher após o período da lactação. Diante do exposto, o relato justifica a desvalorização do seio como objeto de amamentação, desconsiderando a fisiologia dos mamíferos ${ }^{22}$.

Nos aspectos econômicos, os relatos colocam em evidencia a situação financeira como um dos principais fatores que podem dificultar o cuidado com a alimentação infantil. 
"A situação financeira deles dificulta nossas ações, são pessoas esclarecidas e que recebem o bolsa família, porém não aplicam na qualidade da alimentação das crianças, eles compram celular, geladeira, e principalmente bebida alcoólica. Tenho até um caso, eu perguntei do que a criança gosta, a mãe disse que ela gostava de maçã, banana, laranja, só não faz é comer porque não tem em casa, mas ela gosta de tudo" (Enfermeiro 01).

"Eu sou altamente contra o bolsa família, eu acho que em raríssimos casos realmente ajuda aquela mãe necessitada, ajuda na alimentação, mas a maioria compra celular, coisas para dentro de casa [...]" (Enfermeiro 02).

Pelo do exposto, percebe-se a magnitude do problema financeiro e sua implicação direta na alimentação infantil. Essa situação revela a dependência financeira governamental, que segundo os relatos, não gera melhora na situação nutricional, visto que existem outras prioridades para os responsáveis pelo recebimento dos recursos provenientes deste programa. Tal carência repercute em menor oferta ou até mesmo em restrição alimentar, podendo gerar vícios alimentares oriundos da introdução de hábitos pouco saudáveis, porém baratos.

Os relatos dos participantes deste estudo, corroboram com a pesquisa sobre impacto e possibilidades futuras do programa de auxílio financeiro governamental a qual ressaltou que ainda não existem estudos, que comprovem a melhoria nutricional das crianças, os pesquisadores destacam que isto é grave, pois o objetivo deste programa é justamente o estado nutricional das crianças beneficiadas ${ }^{23}$.

\section{CONCLUSÃO}

Este estudo constatou, a partir de relatos dos enfermeiros que trabalham na atenção básica, as facilidades no cotidiano para o cuidado com a alimentação infantil. Estas, são relacionadas a fatores condicionantes vinculados à criança e sua família, ao enfermeiro e à unidade básica de saúde. As principais alavancas potencializadoras estão relacionadas ao papel cuidador da mãe; a estrutura financeira da família; a participação ativa dos enfermeiros no engajamento no processo de cuidar da criança na atenção básica; maior compreensão das ações e responsabilidades da atenção básica na perspectiva da promoção da saúde e prevenção de doenças e a acessibilidade das unidades básicas de saúde.

Compreender estas facilidades e os fatores condicionantes, contribui para o exercício da enfermagem na atenção básica, para ações de enfermagem que respeite a singularidade da situação de cada universo seja ele família, enfermeiro ou unidade básica de saúde.

Por outro lado, as dificuldades, neste contexto estão relacionadas a fatores, sociais, culturais e econômicas da criança e/ou de sua família, as quais devem ser igualmente compreendidas no seu universo singular como requisitos fundamentais para que se proporcionem cuidados efetivos no processo de alimentação da criança e que representam importantes entraves no progresso deste cuidado

Nesse contexto, ressalta-se o papel no enfermeiro no reconhecimento e compreensão desses fatores que facilitam e dificultam as práticas do cuidado nos cenários da prática profissional estudados. Por fim, destaca-se a importância do cuidado de Enfermagem para a promoção da saúde da criança, repercutindo em melhor qualidade de vida e distanciamento de agravos nutricionais. Sugerem-se então novos estudos com o intuito de desvelar ações que otimizem este cuidado por meio dos avanços e que minimizem as dificuldades encontradas neste estudo.

\section{REFERÊNCIAS}

1. Ribeiro MO, Sigaud CHS, Rezende MA, Veríssimo MLÓR. Desenvolvimento infantil: a criança nas diferentes etapas de sua vida. In: Fujimore E, Ohara CVC. Enfermagem e a saúde da criança na atenção básica. Barueri: Manole; 2009. p. 61-120. 2. Fidelis CMF, Osório, MM. Consumo alimentar de macro e micronutrientes de crianças menores de cinco anos no Estado de Pernambuco. Rev Bras Saúde Mater Infant. 2007; 7(1): 63-74.

3. Saldiva SRDM, Escuder MM, Mondini L, Levy RB, Venancio SI. Prática alimentar de crianças de 6 a 12 meses e fatores maternos associados. J Pediatr. 2007; 83(1): 53-67. 
4. Batista Filho M, Souza Al, Miglioli TC, Santos MC. Anemia e obesidade: um paradoxo da transição nutricional brasileira. Cad Saude Publica. 2008; 24(2): 247-57.

5. Rocha L, Gerhardt TE, Santos DL. Social heterogeneity and children's nutrition in the rural environment. Rev Lat.-Am Enfermagem. 2007; 15(n.esp.):828-36.

6. Frota MA, Barroso MGT. Repercussão da desnutrição infantil na família. Rev LatAm Enfermagem. 2005; 13(6):996-1000. 7. Etges BI. Abordagem nutricional do pré-escolar, escolar e adolescente. In: Marques BB, Reis MS, Morais RB, organizadores. Estratégias de atenção à saúde da criança e do adolescente. Santa Cruz do Sul: Ed. EDUNISC; 2008. p. 76-85.

8. Ministério da Saúde (BR). Portaria n 2.488 de 21 de outubro de 2011. Dispõe sobre a Política Nacional de Atenção Básica, estabelecendo a revisão de diretrizes e normas para a organização da Atenção Básica, para a Estratégia Saúde da Família (ESF) e o Programa de Agentes Comunitários de Saúde (PACS). Brasília: Ministério da Saúde, 2011.

9. Ministério da Saúde (BR). Política Nacional de atenção básica. Departamento de atenção à saúde. Brasília: Ministério da Saúde, 2006.

10. Barreto JMO, Nery IS, Costa MSC. Estratégia Saúde da Família e internações hospitalares em menores de 5 anos no Piauí, Brasil. Cad. Saúde Pública. 2012; 28 (3): 515-526.

11. Ministério da Saúde (BR). Protocolo de enfermagem na atenção básica de saúde e ambulatórios. Prefeitura Municipal de Teresina-PI, 2012.

12. Moura MAP, Rocha SS. Dinâmica da Assistência de Enfermagem no cuidado à criança na atenção básica. Nursing. 2012; 167 (14): 221-6.

13. Alberti V. Manual de história oral. $3^{\mathrm{a}}$ edição. Rio de Janeiro: Editora FGV; 2005.

14. Boff L. Saber cuidar: ética do humano - compaixão pela terra. 16ed. Petrópolis,RJ: Vozes;1999.

15. Rosa R, Monticelli M, Martins FE, Siebert ERC, Gasperi BL, Martins MN. Mãe e filho: primeiros laços de interação. Esc Anna Nery. 2010; 14(1):105-12.

16. Ministério da Saúde (BR). Manual para a utilização da Caderneta de Saúde da Criança. Departamento de atenção à saúde. Brasília: Ministério da Saúde; 2005.

17. Vieira VL, Sousa JMP, Cervanto-Mancuso AM. Insegurança alimentar, vínculo mãe-filho e desnutrição infantil em áreas de alta vulnerabilidade social. Rev Bras Saúde Matern Infant. 2010; 10(2):199-207.

18. Soares SM, Amaral MA, Silva LA, Silva PAB. Oficinas sobre sexualidade na adolescência: revelando vozes, desvelando olhares de estudantes do ensino médio. Esc Anna Nery. 2008; 12(3): 485-19. Ronzani TM, Silva CM. O programa Saúde da Família segundo profissionais de saúde, gestores e usuários. Cienc Saúde Coletiva. 2008; 13(1):23-34.

20. Martins CBG, Jorge MHPM. Negligência e abandono de crianças e adolescentes: análise dos casos notificados em município do Paraná, Brasil. Pediatria. 2009; 31(3):186-97.

21. Silva IMD, Silva KV, Leal LP, Jarvoski M. Técnica de amamentação: preparo das nutrizes atendidas em um hospital escola, Recife-PE. Rev Rene. 2011; 12(n. esp.):1021-7.

22. Silva MBC, Moura MEB, Silva AO. Desmame precoce: representações sociais de mães. Rev Eletr Enf. 2007; 9(1): 31-50. 23. Soares S, Sátyra N. O programa bolsa família: desenho institucional, impacto e possibilidades futuras. Brasília: Instituto de pesquisa econômica aplicada; 2009.

Recebido em: 18 de fevereiro de 2014. Aprovado em: 22 de maio de 2015. 